\title{
Interaction of Rickettsia felis with histone H2B facilitates the infection of a tick cell line
}

\section{Correspondence}

Kevin R. Macaluso

kmacaluso@vetmed.Isu.edu

Received 10 May 2010

Revised 7 June 2010

Accepted 10 June 2010

\author{
Chutima Thepparit, ${ }^{1}$ Apichai Bourchookarn, ${ }^{3}$ Natthida Petchampai, ${ }^{1}$ \\ Steven A. Barker ${ }^{2}$ and Kevin R. Macaluso ${ }^{1}$
}

\author{
${ }^{1}$ Department of Pathobiological Sciences, Louisiana State University, School of Veterinary \\ Medicine, Baton Rouge, LA 70803, USA \\ ${ }^{2}$ Department of Comparative Biomedical Sciences, Louisiana State University, School of Veterinary \\ Medicine, Baton Rouge, LA 70803, USA \\ ${ }^{3}$ Department of Technology and Industries, Faculty of Science and Technology, Prince of Songkla \\ University, Pattani 94000, Thailand
}

\begin{abstract}
Haematophagous arthropods are the primary vectors in the transmission of Rickettsia, yet the molecular mechanisms mediating the rickettsial infection of arthropods remain elusive. This study utilized a biotinylated protein pull-down assay together with LC-MS/MS to identify interaction between Ixodes scapularis histone H2B and Rickettsia felis. Co-immunoprecipitation of histone with rickettsial cell lysate demonstrated the association of $\mathrm{H} 2 \mathrm{~B}$ with $R$. felis proteins, including outer-membrane protein $\mathrm{B}(\mathrm{OmpB})$, a major rickettsial adhesin molecule. The rickettsial infection of tick ISE6 cells was reduced by approximately $25 \%$ via RNA-mediated H2B-depletion or enzymic treatment of histones. The interaction of $\mathrm{H} 2 \mathrm{~B}$ with the rickettsial adhesin OmpB suggests a role for $\mathrm{H} 2 \mathrm{~B}$ in mediating $R$. felis internalization into ISE6 cells.
\end{abstract}

\section{INTRODUCTION}

Ticks serve as biological vectors for a variety of diseasecausing agents, including protozoa, viruses and bacteria. Unique among the tick-borne bacteria is the close association between ticks and members of the genus Rickettsia. Ixodid ticks can serve as a horizontal transmission vector for particular species such as Rickettsia rickettsii, the aetiological agent of Rocky Mountain spotted fever, or as a vertical transmission reservoir for rickettsial endosymbionts such as Rickettsia peacockii. While rickettsiae vary in their pathogenicity to vertebrate and tick hosts, their requirement for the arthropod vector is unequivocal; however, the infection process within the arthropod remains poorly defined.

In contrast to the dearth of knowledge of tick-Rickettsia interactions, the molecular interaction between rickettsiae and vertebrate cells has been steadily investigated. Studies examining rickettsial attachment to, and invasion of, vertebrate cells in vitro have identified receptor-mediated endocytosis as the mechanism for rickettsial infection. Prior to fusion of the endosome and lysosome, rickettsiae utilize phospholipase D and haemolysin to escape from the endosome (Whitworth et al., 2005). Once in the cytosol,

Abbreviations: Co-IP, co-immunoprecipitation; $\mathrm{CpB}$, carboxypeptidase B; PPlase, peptidyl-prolyl cis-trans isomerise; $\mathrm{qPCR}$, quantitative real-time PCR; RNAi, RNA-mediated interference.

A supplementary figure is available with the online version of this paper. rickettsiae replicate (Weiss, 1982) and some species invade the nucleus (Silverman \& Wisseman, 1979). Successive infections occur by host cell lysis and the infection of new cells, or via recruitment of host-derived actin and the assemblage of actin tails that facilitate the intracellular movement of rickettsiae through the cytoplasm, across the cell membrane and into neighbouring cells (Hackstadt, 1996; Heinzen et al., 1993, 1999). More recent studies have utilized a membrane protein-protein pull-down assay to illuminate the molecular mechanisms of rickettsial infection in vertebrate cells, identifying the vertebrate molecule $\mathrm{Ku} 70$, a subunit of a DNA-dependent protein kinase, as a host cell receptor for Rickettsia conorii, and demonstrating the cascade process of $R$. conorii internalization into nonphagocytic mammalian cells, comprising receptor binding, signal transduction and cytoskeletal rearrangement (Martinez et al., 2005). Specifically, the adherence of $R$. conorii outer-membrane protein $\mathrm{B}(\mathrm{OmpB})$ to $\mathrm{Ku} 70$ on Vero cells stimulates the ubiquitination of Ku70 by ubiquitin ligase. Subsequent signal transduction activates the Arp2/3 complex, coordinating actin rearrangement and the resultant bacterial endocytosis (Martinez et al., 2005; Martinez \& Cossart, 2004).

A number of tick-derived molecules have been identified as being responsive to rickettsial infection (Macaluso et al., 2006). Using a number of techniques, including differential display PCR (Macaluso et al., 2003), subtractive suppression hybridization (Mulenga et al., 2003) and homologue 
cloning (Simser et al., 2004), the constituents of the tickRickettsia interchange are being identified. With the advent of tools including tick-derived cell culture and low-passage rickettsial isolates, further opportunities to examine many of these interactions in vitro now exist (Munderloh \& Kurtti, 1995). In the current study, a pull-down assay was utilized to identify molecules that associate with rickettsiae. Subsequently, RNA-mediated interference (RNAi) and infection bioassays were employed to characterize interactions between ticks and rickettsial molecules. Elucidating the molecular relationship between arthropods and rickettsiae is essential in deciphering the basis of vector competence, transmission events, and the epidemiology of emerging and re-emerging rickettsial diseases.

\section{METHODS}

Tick cell and Rickettsia cultivation. Ixodes scapularis-derived ISE6 cells were cultivated in L-15B medium (Gibco-BRL) supplemented with $10 \%$ fetal bovine serum (FBS) (HyClone) and $10 \%$ tryptose phosphate broth (Sigma), pH 6.8-7.0, with $5 \% \mathrm{CO}_{2}$ at $32{ }^{\circ} \mathrm{C}$ (Munderloh \& Kurtti, 1989). Rickettsia felis (LSU strain) was propagated in ISE6 cells as previously described (Pornwiroon et al., 2006) and harvested when the infected cells were around $80-90 \%$ confluent. For rickettsial infection inhibition assays, low-passage $R$. felis cultures were partially purified as described by Sunyakumthorn et al. (2008). For pull-down and co-immunoprecipitation assays, Rickettsia cells were purified by Renografin density-gradient centrifugation to remove host-derived protein contamination as described previously (Pornwiroon et al., 2009). Rickettsial viability and enumeration were assessed as previously described (Sunyakumthorn et al., 2008).

Pull-down assay. ISE6 cells were surface-labelled with Sulfo-NHS-LCBiotin (Pierce) for $30 \mathrm{~min}$ on ice. The biotinylated cells were sonicated in ice-cold $0.1 \%$ NP-40/PBS buffer. The insoluble materials were discarded after centrifugation and the protein extracts in the soluble fraction were incubated with Renografin-purified $R$. felis for $12 \mathrm{~h}$ at $4{ }^{\circ} \mathrm{C}$. Rickettsia-bound protein pellets were collected, washed, and eluted using $0.1 \% \mathrm{NP}-40 / \mathrm{PBS}$ with $1 \mathrm{M} \mathrm{NaCl}$ for $1-1.5 \mathrm{~h}$ at $4{ }^{\circ} \mathrm{C}$. Eluates were analysed on Bistris gels (Invitrogen) with Western blotting analysis using peroxidase-conjugated streptavidin. For a negative control, Escherichia coli (Invitrogen TOP10 E. coli strain, similar to the DH10B strain) was used in the assays instead of purified $R$. felis. Grown in LB broth for $16 \mathrm{~h}$, E. coli was collected by centrifugation at $3300 \mathrm{~g}$ for $10 \mathrm{~min}$, washed twice with PBS and then used in the pull-down assays.

Protein identification. The protein bands of interest were identified by LC-MS/MS as previously described (Sunyakumthorn et al., 2008). Searches were performed in the NCBInr and Swiss-Prot databases using a Mascot search via http://www.matrixscience.com.

Gel electrophoresis and Western blotting. Cell lysates or protein extracts were subjected to electrophoresis through either $4-12 \%$ or $12 \%$ Bistris gels and transferred to a PVDF membrane. The membrane was blocked with $5 \%$ skim milk in TBS-T at room temperature for $1 \mathrm{~h}$. Primary antibodies were diluted 1:5000 for $\alpha$ histone H2B (ab1790; Abcam) and 1: 400 for $\alpha$-tubulin (B-5-1-2) (sc23948, Santa Cruz Biotechnology). HRP-conjugated secondary antibodies, goat anti-rabbit IgG (A-6154, Sigma), and goat antimouse IgG (Pierce) were used at 1:10000 dilution. The signal was developed by using a chemiluminescent substrate kit (Pierce).

Quantitative real-time PCR (qPCR). The copy numbers of rickettsial and tick genes were established by $\mathrm{qPCR}$ as recently published (Zanettii et al., 2008). Infectivity was quantified in terms of rickettsiae per host cell by a qPCR using specific primers for the single copy of the rickettsial $17 \mathrm{kDa}$ antigen gene and the tick calreticulin gene. A plasmid containing portions of both genes was constructed as described by Zanettii et al. (2008), and used as a standard for template quantification. The percentage of infection was determined designating the infectivity (the quantity of rickettsiae per host cells) of the control group as $100 \%$.

RNA-mediated interference of tick gene transcription. dsRNA specific to the full-length gene for tick histone $\mathrm{H} 2 \mathrm{~B}$ was synthesized from ISE6 RNA by RT-PCR. Synthesized from ISE6 total RNA, the full-length gene for histone $\mathrm{H} 2 \mathrm{~B}$ was amplified from the first-strand cDNA by using a pair of specific primers containing the T7 promoter sequence at the $5^{\prime}$ end. Primer sequences are $5^{\prime}$-TTA ATA CGA CTC ACT ATA GGG CGC ACG TCC GAC AAG AAG AAG AAA-3' and 5'-TTA ATA CGA CTC ACT ATA GGG TTG AAG AAG TGT ACT TCG TGA CCG-3'. The PCR product was transcribed into dsRNA based on the T7 RNA polymerase promoter sequence by using a MEGAscript T7 kit (Ambion). Scramble dsRNA was synthesized, using a pTRI-Xef DNA template provided in the kit, for use as a negative control. dsRNA specific to the gene for histone $\mathrm{H} 2 \mathrm{~B}$ or scramble dsRNAs were introduced into ISE6 cells by using RNAiFect transfection agent (Qiagen). In parallel, ISE6 cells were transfected with fluorescently labelled dsRNA (Cy3 siRNA Labelling kit, Ambion) to assess the transfection efficiency and subcellular localization of dsRNA. To determine the post-transfection period at which the targeted protein expression was depleted, the transfected cells were collected daily to assess the regulation of gene transcription, by RTPCR, and protein expression, by Western blotting.

Infection inhibition assays. ISE6 cells grown on 48-well plates were transfected with or without $14 \mathrm{nM}$ dsRNAs targeting histone $\mathrm{H} 2 \mathrm{~B}$ or scramble genes. The infection inhibition assay was performed on the day post-transfection on which the reduction of the targeted protein was observed as described earlier. The treated cells were infected with partially purified $R$. felis at an m.o.i. of 200 at $32{ }^{\circ} \mathrm{C}$ for $90 \mathrm{~min}$. Then unbound $R$. felis was removed and the infected cells were washed twice with PBS prior to collection. Genomic DNA was extracted from the samples to assess the rickettsial and tick gene number by qPCR. The assays were performed in triplicate. For carboxypeptidase B $(\mathrm{CpB})$ treatment, ISE6 cells were pre-treated with 0,10 , or 50 units of $\mathrm{CpB}$ (Worthington) in serum-free L-15B medium at $37^{\circ} \mathrm{C}$ for 60 min prior to infection with $R$. felis.

Co-immunoprecipitation (Co-IP). Histones were extracted from ISE6 cells by lysis with Triton Extraction Buffer (TEB: PBS containing $0.5 \%$ Triton X-100 and protease inhibitor) on ice for $10 \mathrm{~min}$ with gentle stirring. Cell pellets were collected, washed with TEB buffer and then incubated with $0.2 \mathrm{M} \mathrm{HCl}$ for $16 \mathrm{~h}$ at $4{ }^{\circ} \mathrm{C}$. Histones in the supernatant fraction were harvested by centrifugation at $380 \mathrm{~g}$ for $10 \mathrm{~min}$ at $4{ }^{\circ} \mathrm{C}$. The Renografin-purified $R$. felis cells were lysed in $1 \% \mathrm{NP}-40$ buffer; the protein was quantified by Bradford assay and $100 \mu \mathrm{g}$ was pre-incubated with $100 \mu \mathrm{g}$ of the acid-extracted histone at $4{ }^{\circ} \mathrm{C}$ overnight. The Rickettsia-H2B complex was captured and insolubilized by incubation with $2 \mu \mathrm{g}$ of polyclonal antibody against histone H2B followed by the antibody-binding protein G (Santa Cruz Biotechnology). The Co-IP products were collected by centrifugation at $600 \mathrm{~g}$ for $5 \mathrm{~min}$ and washed in $1 \% \mathrm{NP}-40$ prior to gel electrophoresis and Western blotting.

Histone-mediated rickettsial entry assay. The partially purified $R$. felis cells were pre-incubated with or without the acid-extracted histone from ISE6 cells $(2-10 \mu \mathrm{g})$ in a total volume of $500 \mu \mathrm{l}$ for $1 \mathrm{~h}$ at $32{ }^{\circ} \mathrm{C}$ prior to infection (m.o.i. of 200) of the ISE6 cells pre-grown on a 96-well tissue culture plate. After infection for $90 \mathrm{~min}$ at $32{ }^{\circ} \mathrm{C}$, rickettsial inocula were removed and the infected cells were collected 
for DNA extraction. Numbers of rickettsiae per host cell were determined by qPCR as mentioned earlier. To assess the role of the C-terminal portion of the histone in rickettsial entry mediation, the extracted histone was pre-treated with 50 units of $\mathrm{CpB}$ at $37^{\circ} \mathrm{C}$ for $60 \mathrm{~min}$. The enzymic reaction was stopped by addition of $\mathrm{HCl}$ to a final concentration of $0.05 \mathrm{M}$. The partially purified $R$. felis cells were incubated with the $\mathrm{CpB}$-treated or untreated histone prior to infecting (m.o.i. of 100) ISE6 cells pre-grown on coverslips. After infection for $90 \mathrm{~min}$ at $32{ }^{\circ} \mathrm{C}$, rickettsial inocula were removed and the Rickettsiaassociated cells were analysed by immunofluorescence assay.

Immunofluorescence assay. Uninfected and $R$. felis-infected ISE6 cells, pre-grown on coverslips, were fixed with $4 \%$ paraformaldehyde in PBS and permeabilized with $0.5 \%$ Triton X-100 in PBS. To detect $R$. felis, the fixed cells were stained with mouse anti-R. felis serum $(1: 200)$, followed by staining with FITC-conjugated anti-mouse IgG antibody $(1: 200, \mathrm{KPL})$. Slides were examined by fluorescence microscopy using the $\times 63$ oil objective.

Statistical analysis. The raw data and ranked data were analysed by one-way ANOVA with repeated measurement, followed by post hoc tests, for which both Tukey's HSD test and the Ryan-Einot-GabrielWelsch test were performed using the SAS GLM procedure. For all comparisons, a $P$-value $\leqslant 0.05$ was taken as significant.

\section{RESULTS}

\section{Binding of $\boldsymbol{R}$. felis to ISE6 cell-surface histone H2B}

A bacterial affinity pull-down assay was employed to assess rickettsial interaction with the ISE6 cell-surface proteins. The cell-surface proteins of intact ISE6 cells were selectively

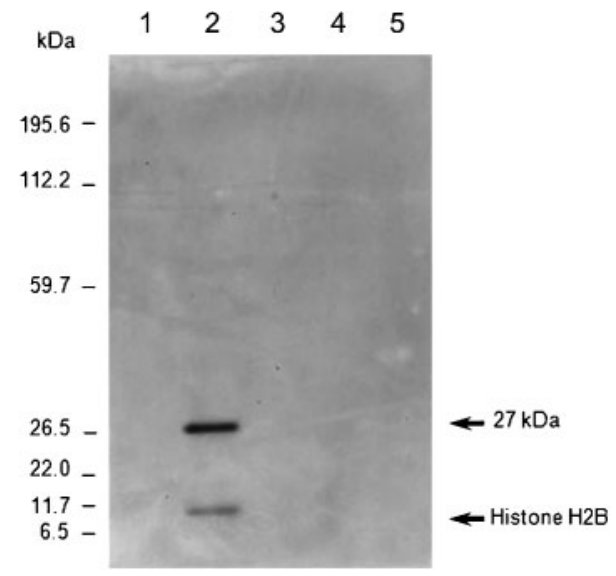

Fig. 1. $R$. felis interacts with ISE6 cell surface proteins. Western blotting analysis of eluates from pull-down assays using biotinylated ISE6 surface proteins and/or Renografin-purified $R$. felis. Biotinylated ISE6 cells in 0.1\% NP-40/PBS buffer were incubated with or without purified $R$. felis. Rickettsia-bound protein pellets were collected, washed, and eluted using $0.1 \% \mathrm{NP}-40 / \mathrm{PBS}$ with $1 \mathrm{M}$ $\mathrm{NaCl}$. Eluates were analysed on Bistris gels with Western blotting analysis using peroxidase-conjugated streptavidin. Lanes 1, labelled ISE6 protein; 2, labelled ISE6 protein $+R$. felis; 3, unlabelled ISE6 protein; 4, unlabelled ISE6 protein $+R$. felis; $5, R$. felis. labelled with biotin prior to incubation with purified $R$. felis. The rickettsia-bound proteins were eluted, and analysed on Bistris gels with Western blotting analysis using peroxidase-conjugated streptavidin. Two putative Rickettsia-binding proteins, with approximate sizes of 14 and $27 \mathrm{kDa}$, specifically bound to purified $R$. felis (Fig. 1). The specificity of the Rickettsia-arthropod interaction was confirmed when the 14 and $27 \mathrm{kDa}$ proteins were not detected when using E. coli instead of Rickettsia as a control for the pull-down assay (Supplementary Fig. S1, available with the online version of this paper). The trypsin-digested peptides of the $14 \mathrm{kDa}$ band analysed by LC-MS/MS significantly matched $I$. scapularis histone H2B (GenBank accession no. AAY66901). Peptide analysis of the $27 \mathrm{kDa}$ band identified a parvulin-like peptidyl-prolyl cis-trans isomerase (PPIase) (GenBank accession no. YP_246958), one of the outer-membrane proteins of $R$. felis. The interaction of $R$. felis with tick histone $\mathrm{H} 2 \mathrm{~B}$ was confirmed by co-immunoprecipitation. Histone extracted from ISE6 cells was incubated with $R$. felis protein lysate prior to incubation with antibody against histone H2B and coupling with antibody-binding protein-G. As shown in Fig. 2, Western blotting analysis of the Co-IP eluates probed with mouse anti- $R$. felis revealed that three bands of $R$. felis proteins coeluted with a histone-Rickettsia complex. Mass-spectometry analysis of trypsin-digested peptides

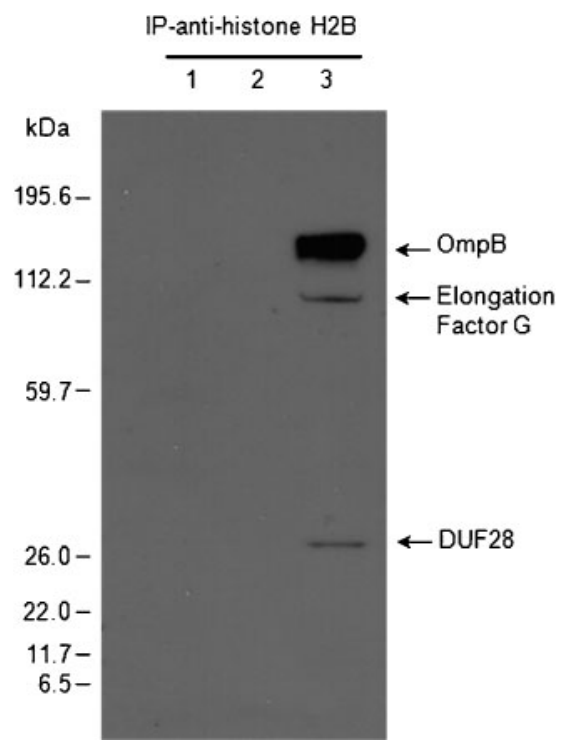

Fig. 2. $R$. felis OmpB protein binds ISE6 histone H2B. Histone extract from ISE6 cells was pre-incubated with or without Renografin-purified R. felis. The Rickettsia-H2B complex was captured and insolubilized by sequential incubation with polyclonal antibody against histone $\mathrm{H} 2 \mathrm{~B}$ and antibody-binding protein $\mathrm{G}$. The Co-IP products were collected and analysed on Bistris gels by Western blotting using mouse anti- $R$. felis serum. Lanes 1, ISE6 histone extract; 2 , purified $R$. felis cell lysate; 3 , lysates of ISE6 histone extract and the purified $R$. felis. Corresponding proteins were identified by LC-MS/MS. 
identified two minor bands, which matched to $R$. felis elongation factor $\mathrm{G}$ (GenBank accession no. Q8KTA8) and the hypothetical protein DUF28 with unknown function (GenBank accession no. YP_246815), and a major band of approximately $120 \mathrm{kDa}$, identified as $R$. felis OmpB (GenBank accession no. AAY61056). The histone H2B band was detected in the Western blotting membrane when reprobed with antibody against $\mathrm{H} 2 \mathrm{~B}$ to confirm the presence of $\mathrm{H} 2 \mathrm{~B}$ in the histone-Rickettsia binding complex (data not shown).

\section{RNA-mediated interference (RNAi) of tick gene transcription}

To further delineate the role of histone $\mathrm{H} 2 \mathrm{~B}$ in the rickettsial infection of tick cells, we utilized RNAi to deplete cells of H2B. In the current study, RNAi methodology was adapted to ISE6 cells to knock down transcription and translation of the putative R. felis-binding molecule histone H2B. ISE6 cells were either mock-transfected with only transfection buffer (negative control), transfected with dsRNA targeting the full-length gene for the tick histone $\mathrm{H} 2 \mathrm{~B}$, or transfected with scrambled nucleotides (nonspecific control). The transfection efficiency and subcellular localization of dsRNA were verified at $24 \mathrm{~h}$ post-transfection by detection of fluorescently labelled dsRNA under fluorescence microscopy (Fig. 3a). The transfected cells were collected daily to assess the gene transcription and protein regulation of the gene/protein of interest. The introduction of 1.4 and $14 \mathrm{nM}$ dsRNA, targeting H2B production specifically, depleted the targeted gene transcription as early as 3 days post-transfection (data not shown), with silencing persisting through to day 11 (Fig. 3b). The knocked-down transcript was recovered at day 13 posttransfection (data not shown). Although the silencing effect at the translational level could not be clearly observed in the early period post-transfection, at day 11 post-transfection a reduction of $\mathrm{H} 2 \mathrm{~B}$ protein in the cells transfected with $14 \mathrm{nM}$ dsRNAs was observed (Fig. 3c). The altered regulation of $\mathrm{H} 2 \mathrm{~B}$ expression was normalized with $\alpha$ tubulin and the band intensity was quantified by densitometry analysis (Fig. 3d). Relative to the mock-transfected control, H2B expression was reduced by approximately $26 \%$ and $15 \%$ in the cells transfected with dsRNA targeting $\mathrm{H} 2 \mathrm{~B}$ at $14 \mathrm{nM}$ and $1.4 \mathrm{nM}$, respectively. The induction of the scramble dsRNA did not affect the regulation of $\mathrm{H} 2 \mathrm{~B}$ at either the transcriptional or the translational level (Fig. 3b, c, d).

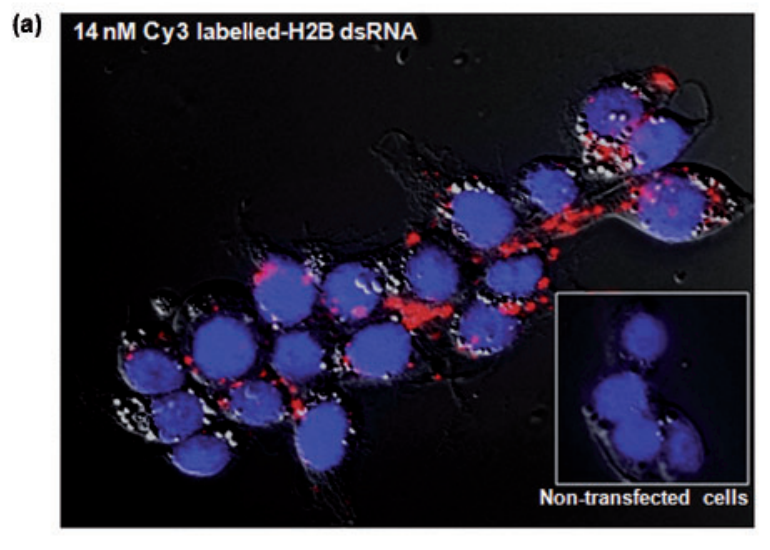

(b)
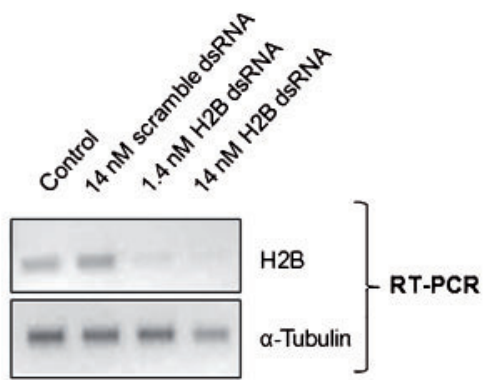

(c)

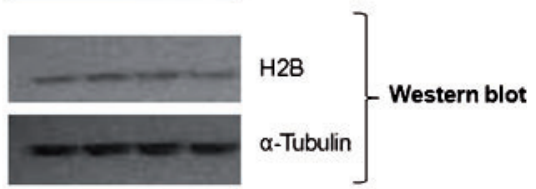

(d)

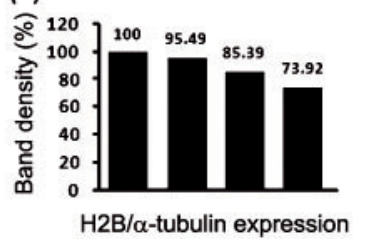

Fig. 3. Transcriptional and translational silencing of tick histone H2B using dsRNA in ISE6 cells. ISE6 cells were transfected with dsRNA as indicated. (a) The transfection efficiency and cellular localization of dsRNA was observed at $24 \mathrm{~h}$ posttransfection by detection of Cy3-labelled dsRNA in the transfected cells under fluorescence microscopy. Cell nuclei were stained with DAPI. Red fluorescence represents localization of dsRNA. At day 11 post-transfection, regulation of histone $\mathrm{H} 2 \mathrm{~B}$ transcripts and protein was observed. (b) RT-PCR detection of histone H2B and $\alpha$-tubulin transcripts of the dsRNA-transfected cells. (c) Western blotting analysis using anti-histone H2B antibody. The membrane was reprobed with anti- $\alpha$-tubulin antibody. (d) Densitometry of histone H2B and $\alpha$-tubulin Western blotting. Band density was analysed using Quantity One analysis software (Bio-Rad). The density of histone H2B was normalized with $\alpha$-tubulin. The bar graph shows the expression of histone $\mathrm{H} 2 \mathrm{~B}$ relative to that of the control $(100 \%)$. 


\section{Histone H2B is involved in the infection of tick cells by $R$. felis}

To determine if the putative $R$. felis-binding protein histone $\mathrm{H} 2 \mathrm{~B}$ is functionally involved in the infection of tick cells, the infectivity of $R$. felis in the dsRNA-mediated-H2B-depleted ISE6 cells was assessed. The reduction of $\mathrm{H} 2 \mathrm{~B}$ expression by dsRNA-mediated silencing inhibited $R$. felis infection by approximately $61 \%$ relative to the control. The infectivity of $R$. felis was variable in the scramble dsRNA-treated cells; however, there was no inhibitory effect on rickettsial infectivity (Fig. 4). In a separate bioassay, the histonemediated infection of tick cells by rickettsiae was assessed. Pre-incubation of partially purified $R$. felis with the histones extracted from ISE6 cells could facilitate rickettsial infection in a dose-dependent manner, as shown by the significantly increased number of infected cells in the histone preincubation groups (Fig. 5).

\section{A carboxy-terminal region of histone H2B is involved in the infection of tick cells by $R$. felis}

Histone $\mathrm{H} 2 \mathrm{~B}$ is rich in basic amino acids lysine and arginine at its $\mathrm{C}$ terminus. C-terminal lysyl residues of $\mathrm{H} 2 \mathrm{~B}$ were previously reported to serve as a plasminogen-binding site (Das et al., 2007; Herren et al., 2006; Miles et al., 1991).

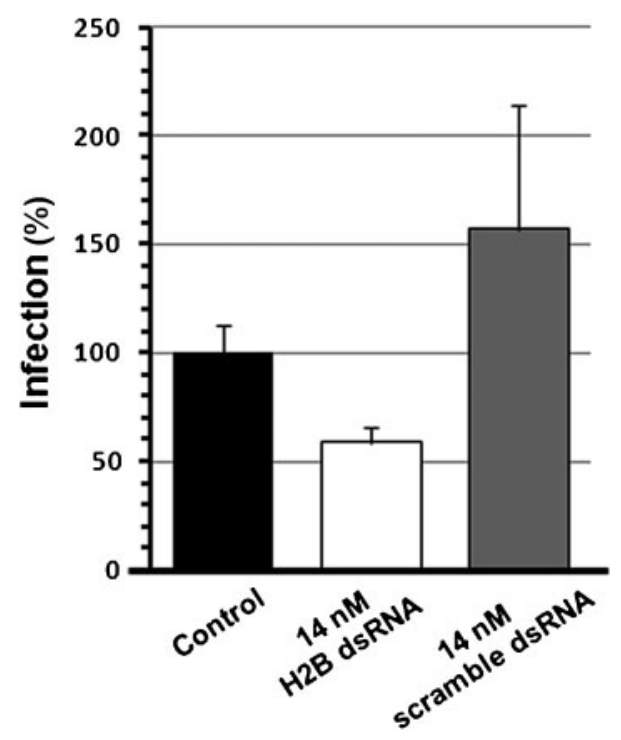

Fig. 4. RNAi-mediated depletion of host cell histone decreases rickettsial infection. The infection bioassay was performed when histone H2B was depleted in ISE6 cells (11 days post-transfection). ISE6 cells (control), histone H2B-depleted cells (14 nM H2B dsRNA), or mock-transfected cells (14 nM scramble dsRNA) were infected (m.o.i. 200) with partially purified $R$. felis for $90 \mathrm{~min}$ at $32{ }^{\circ} \mathrm{C}$. Unbound $R$. felis was removed and infected cells were washed with PBS prior to collection. Infectivity was calculated (rickettsial gene/host gene) by qPCR and is shown as a percentage of the control value. Bars represent the mean ( \pm SEM) of three individual assays.

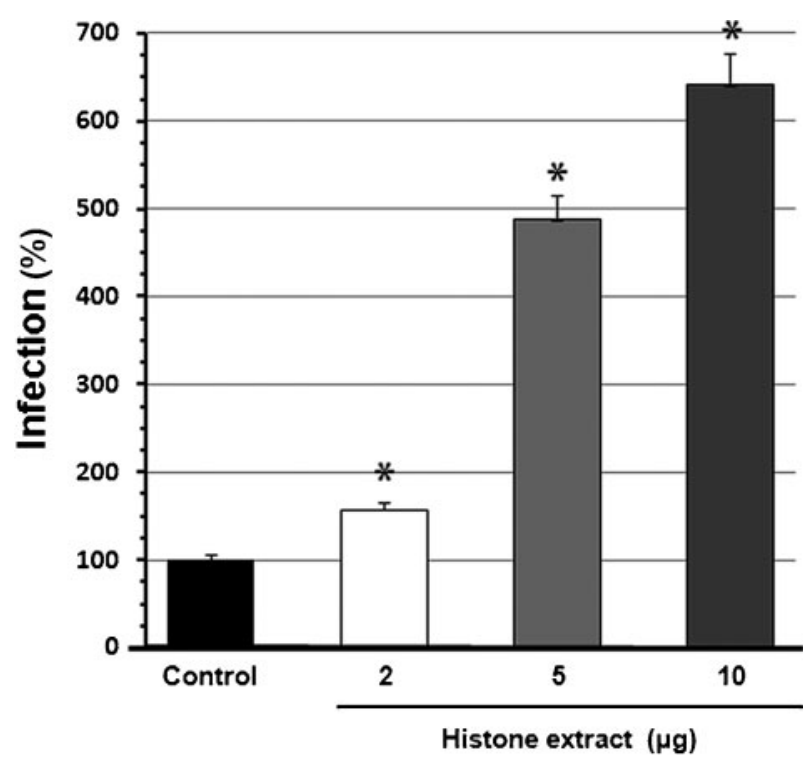

Fig. 5. Histone enhances $R$. felis entry into tick cells. Partially purified $R$. felis was pre-incubated with tick-derived histone (2$10 \mu \mathrm{g}$ ) for $1 \mathrm{~h}$ at $32{ }^{\circ} \mathrm{C}$ prior to infection (m.o.i. 200) of ISE6 cells. After $90 \mathrm{~min}$, rickettsial inocula were removed and cells were collected. Infectivity was calculated (rickettsial gene/host gene) by qPCR. The bar graph represents percentage of infection relative to the control (as $100 \%)$. Bars represent the mean ( \pm SEM) of three individual assays. Asterisks represent a significant difference from the control.

To determine if the C-terminal basic amino acid region of H2B is involved in $R$. felis infection, ISE6 cells were treated with $\mathrm{CpB}$ to remove the basic amino acids, including lysine, arginine and ornithine, from the $\mathrm{C}$ terminus of polypeptides prior to infection with $R$. felis. The effectiveness of $\mathrm{CpB}$ in cleavage of the $\mathrm{C}$-terminal region of histone H2B on ISE6 cells was determined by Western blotting with antibody raised against the $\mathrm{C}$ terminus of histone $\mathrm{H} 2 \mathrm{~B}$. Of the varying $\mathrm{CpB}$ concentrations used, a saturation effect was observed on the ISE6 cells treated with 50 units of $\mathrm{CpB}$ as shown by the least amount of $\mathrm{H} 2 \mathrm{~B}$ remaining intact (Fig. 6a). Increasing $\mathrm{CpB}$ concentration to 100 units did not result in additional cleavage of $\mathrm{H} 2 \mathrm{~B}$; therefore, the infection inhibition assay was done with the ISE6 cells treated with or without 10 or 50 units of $\mathrm{CpB}$. The infectivity of $R$. felis in the CpB-treated ISE6 cells was compared to the control cells. Fig. 6(b) shows a dosedependent inhibitory effect of $\mathrm{CpB}$ treatment on rickettisal infection. The C-terminal portion of the histone contributes to rickettsial infection, as $\mathrm{CpB}$ treatment of the ISE6 cells inhibited $R$. felis infection by approximately $10 \%$ and $25 \%$ relative to the control for 10 and 50 units, respectively. To determine if the inhibitory effect of $\mathrm{CpB}$ treatment is specific to the $\mathrm{C}$-terminal lysine residues of histone, resulting in rickettsial infection inhibition, histone was treated with 50 units $\mathrm{CpB}$ to cleave the C-terminal basic amino acids before incubation with the partially 
(a)

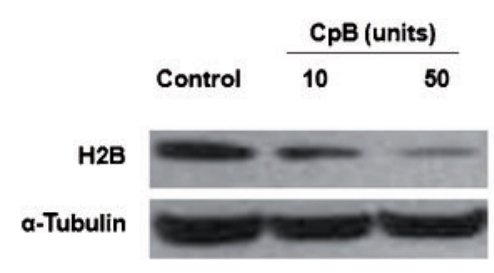

(c)

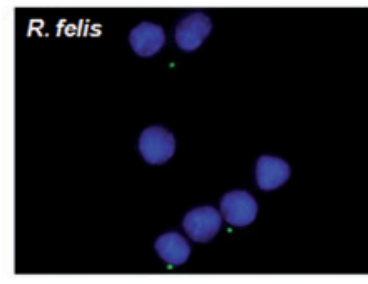

(b)

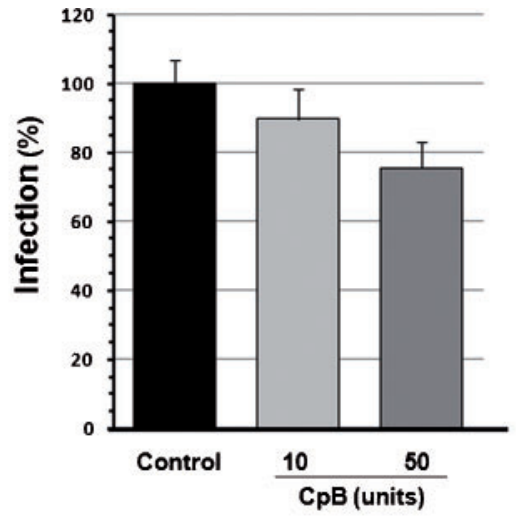

Histone + R. felis

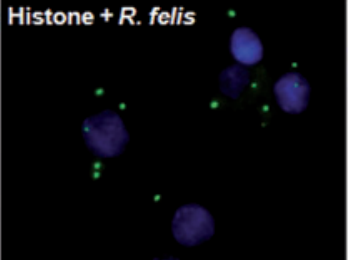

CpB-treated histone $+R$. felis
Fig. 6. Carboxypeptidase treatment decreases rickettsial infection. ISE6 cells were pretreated with various concentrations of $\mathrm{CpB}$ at $37{ }^{\circ} \mathrm{C}$ for $1 \mathrm{~h}$ prior to infection (m.o.i. 200) with partially purified $R$. felis for $1 \mathrm{~h}$ at $32{ }^{\circ} \mathrm{C}$. (a) The effectiveness of $\mathrm{CpB}$ in cleavage of $\mathrm{C}$ terminal histone $\mathrm{H} 2 \mathrm{~B}$ on ISE6 cells was determined by Western blotting analysis using anti-histone $\mathrm{H} 2 \mathrm{~B}$ antibody with anti- $\alpha$-tubulin antibody as a load control. (b) Infectivity of Rickettsia on the $\mathrm{CpB}$ treated/untreated ISE6 cells as calculated (rickettsial gene/host gene) by qPCR. The bar graph represents percentage infection relative to the control (as 100\%). Bars represent \pm SEM of three individual assays. (c) ISE6 cells were infected (m.o.i. $100)$ with $R$. felis alone, $R$. felis pre-incubated with $10 \mu \mathrm{g}$ histone, or $R$. felis pre-incubated with 50 units $\mathrm{CpB}$-treated histone. After $90 \mathrm{~min}$, inocula were removed and cells were fixed and permeabilized. Rickettsiae were detected by immunofluorescence assay and cell nuclei were stained with DAPI. Lower numbers of rickettsiae were observed in the CpB-treated preparations. purified R. felis. Either R. felis alone or R. felis with CpBtreated/untreated histone were incubated with ISE6 cells and infected cells were analysed by immunofluorescence assay (Fig. 6c). Corresponding to the ability of histone to mediate rickettsial entry (Fig. 5), a marked accumulation of rickettsiae in the histone pre-incubation group was observed compared to $R$. felis alone. Rickettsial accumulation declined in the $\mathrm{CpB}$-treated histone pre-incubation group suggesting that the $\mathrm{C}$-terminal basic amino acid residues serve as a Rickettsia-binding region or participate in mediation of rickettsial entry.

\section{DISCUSSION}

The initial interaction between Rickettsia and the arthropod host is a critical determinant of successful rickettsial infection and transmission; however, progress made in identifying host molecules facilitating or involved in rickettsial infection is lacking. This study thus sought to identify and characterize arthropod-derived molecules involved in the rickettsial infection of arthropod host cells. The utilization of the pull-down assay was intended to identify arthropod-derived proteins that bind rickettsiae. The specificity of the reaction was verified by a control reaction using E. coli, which did not result in the identification of the same bands. Although $R$. felis is typically associated with insect hosts, there is molecular evidence of $R$. felis in acarine hosts such as ixodid ticks (reviewed by Reif \& Macaluso, 2009). The isolation of $R$. felis (strain LSU) in the I. scapularis (ISE6) cell line allows assessment of a low-passage rickettsial isolate; however, the association of identified molecules should be confirmed in Rickettsia-arthropod relationships observed in nature.

Interestingly, the rickettsial parvulin-like PPIase protein produced a detectable streptavidin/biotin signal in the pulldown assay. The occurrence of rickettsial parvulin-like PPIase in the tick-Rickettsia pull-down eluates suggests an association of this protein with the Rickettsia-host cell surface protein interaction. Although the identification of a parvulin-like PPIase was unanticipated, it generates interest regarding potential functional activity in rickettsial infection. Bacterial PPIase-containing proteins have been found to be involved in protein trafficking, arrangement or maturation of outer-membrane proteins, and bacterial virulence (Behrens et al., 2001; Emelyanov \& Demyanova, 1999). With respect to bacterial infection, it was proposed that parvulin-like PPIases may alter the conformation of either bacterial or host cell surface components, facilitating adherence between bacteria and host cells (Emelyanov \& Demyanova, 1999). The involvement of PPIase-containing proteins in host cell adherence was clearly demonstrated in other bacterial infections. For example, inactivation of the PPIase domain of the Mip protein of Legionella pneumophila, and the Mip-like protein of Chlamydia trachomatis, both of which share similar PPIase activity, interfered with the initiation of bacterial infection of mammalian cells (Lundemose et al., 1993). Although not of tick origin, the detection of this rickettsial outer-membrane protein, possessing putative adhesion activity in the host cell binding complex, suggests it has a role in the Rickettsiahost interaction, and that it is of interest for further investigation. 
A tick-derived molecule, histone $\mathrm{H} 2 \mathrm{~B}$, was also identified via the Rickettsia and tick cell surface protein interaction pull-down assay. Histone $\mathrm{H} 2 \mathrm{~B}$, one of the core histones forming the nucleosome complex with the linker histone $\mathrm{H} 1$, primarily locates and functions in the cell nucleus (Baake et al., 2001; Khan et al., 1998). However, consistent with the current study, there is increasing evidence that histone proteins localize in other cellular compartments, for example in the cytoplasm and on the cell surface of both eukaryotic and prokaryotic cells (Bolton \& Perry, 1997; Das et al., 2007; Herren et al., 2006; Holers \& Kotzin, 1985; Khan et al., 1998; Qiu \& Wang, 2008; Zlatanova et al., 1990). Histone H2B lacks a transmembrane domain (Herren et al., 2006); a clear translocation process from nucleus to cell membrane has not been determined. However, histone $\mathrm{H} 2 \mathrm{~B}$ in the form of DNA-core histone complexes has been identified on activated T-cell membranes anchoring to a heparan sulfate proteoglycan (Watson et al., 1999). The association between a tickderived nuclear protein such as histone $\mathrm{H} 2 \mathrm{~B}$ and rickettsiae is not surprising, as evidence of the receptor function of typical non-cell surface proteins is increasing. For example, $\mathrm{Ku} 70$ and its heterodimer, $\mathrm{Ku} 80$, are typically retained in the nucleus and play several roles, including the repair of double-strand breaks by joining the DNA ends, facilitating the coordination of other DNA repair proteins (Featherstone \& Jackson, 1999). In an undefined process, Ku70 translocates from the nucleus to the cytoplasm and also to the plasma membrane, where it is multi-functional (Amsel et al., 2008; Prabhakar et al., 1990; Tai et al., 2002).

Rickettsial invasion initiates with the bacteria binding a molecule(s) on the cell surface of the host cell. The Co-IP results demonstrated the interaction of rickettsial proteins with tick histone $\mathrm{H} 2 \mathrm{~B}$. Among these proteins, three rickettsial antigenic proteins were recognized by mouse anti-R. felis; these include elongation factor $\mathrm{G}$, the hypothetical protein DUF28, and OmpB. OmpB, the most abundant immunogenic surface protein of Rickettsia, is one of the crystalline bacterial cell surface layer (S-layer) proteins, with known functions in cell adhesion and surface recognition (Zlatanova et al., 1990) in vertebrates, and specific interaction with the mammalian DNAbinding molecule Ku70. Although outer-membrane proteins are antigenically specific among various rickettsial species (Anacker et al., 1987), the critical role in host cell invasion seems conserved (Li \& Walker, 1998; Martinez et al., 2005; Uchiyama et al., 2006). Other rickettsial molecules have been identified as adhesins or are associated with infection, including outer-membrane protein A (OmpA). The gene encoding OmpA is present in $R$. felis and is commonly found in the members of the spotted fever group of Rickettsia; however, R. felis ompA possesses premature stop codons (Zavala-Castro et al., 2005). The truncated ompA gene encodes the putative OmpA protein, which lacks the $\beta$-barrel autotransporter fragment, resulting in the inability to export molecules to the outer membrane (Zavala-Castro et al., 2005). Rickettsial infection despite a nonfunctional OmpA suggests that redundant mechanisms of rickettsial adhesion to the host cell exist.

Effectively applied RNAi can facilitate the study of proteins in vivo and in vitro for several species of tick (Kurtti et al., 2008; Ramakrishnan et al., 2005). In the current study, an inhibitory effect on $R$. felis infection was observed in the absence of a complete knock-down of histone H2B protein. RNA-mediated interference has been effectively applied to study the functions of several proteins in various cells, albeit with limited effectiveness for some proteins. While dsRNA targeting H2B could efficiently and immediately knock down histone mRNA, the $\mathrm{H} 2 \mathrm{~B}$ protein was not completely depleted. The impaired effect of dsRNAmediated silencing at the protein level might occur because of a low turnover rate for the histone protein. Although histone mRNA reportedly has a half-life as short as $10 \mathrm{~min}$ to several hours (Borun et al., 1967; Marzluff \& Duronio, 2002), the rate of histone protein turnover was estimated to be relatively slow [estimated half-life $30 \mathrm{~h}$ : computational analysis by ProtParam (Gasteiger et al., 2005)]. In addition, histone protein is abundantly synthesized and accounts for $30 \%$ of total protein synthesis (Kedes, 1979). Consistent with the results of the current study, the delayed effect of RNAi on protein expression in tick cell culture (ISE6) was also noted by Kurtti et al. (2008), in which decreases in detectable protein expression began on day 7 with the lowest levels of expression detected at day 21 posttransfection. Likewise, in another I. scapularis cell line, IDE8, while some of targeting genes were partially silenced by RNAi, the silencing effect on protein level was not provided (de la Fuente et al., 2007). Compared to in vivo RNAi in ticks (Ramakrishnan et al., 2005), it is not clear if the mechanisms of RNAi are slow due to the target molecule, or due to the origin of the host cell (cell culture versus whole tick).

The specificity of histone $\mathrm{H} 2 \mathrm{~B}$ residues participating in rickettsial binding was further verified via enzymemediated histone modification. Histone $\mathrm{H} 2 \mathrm{~B}$ possesses a basic amino-acid-rich region at its $\mathrm{C}$ terminus, composed of lysine and arginine residues. C-terminal lysyl residues of $\mathrm{H} 2 \mathrm{~B}$ were demonstrated to serve as a plasminogen recognition binding site (Das et al., 2007; Herren et al., 2006; Miles et al., 1991) and the blocking of H2B with an antibody specific to its C-terminal octopeptide, including the cleavage of its C-terminal lysyl residues by $\mathrm{CpB}$, abolished the binding of plasminogen to the cells (Herren et al., 2006). The loss of infectivity of rickettsiae on the CpB-treated ISE6 cells implies the involvement of these Cterminal, positively charged residues of $\mathrm{H} 2 \mathrm{~B}$ in Rickettsia binding. Interestingly, the lysine-rich $\mathrm{C}$-terminal region was also reported as the binding region on histone $\mathrm{H} 1$, which serves as the specific receptor for the adhesin of enterotoxigenic E. coli (Zhu et al., 2005). The involvement of H2B in the rickettsial infection of arthropod host cells was confirmed in vitro by different approaches. The study 
demonstrates that in ISE6 cells the partial depletion of $\mathrm{H} 2 \mathrm{~B}$ by RNAi inhibits rickettsial infection and the cleavage of the $\mathrm{C}$-terminal region of $\mathrm{H} 2 \mathrm{~B}$ by $\mathrm{CpB}$ limits histone-mediated infection. Although no single method completely abrogated the activity of H2B in tick cells, the partial inhibition of infection observed in both bioassays demonstrates a role for $\mathrm{H} 2 \mathrm{~B}$ in the infection of arthropod cells by $R$. felis, suggesting redundant mechanisms of infection are involved. Additionally, the ability of histone to mediate rickettsial entry into the cells confirms the Rickettsia-histone interaction and supports the involvement of histone in rickettsial infection. While we have demonstrated that H2B interacts directly with $R$. felis and facilitates rickettsial infection, we cannot exclude the potential role of histones in serving as an intermediary between rickettsiae and an additional host factor. The fact that the addition of increasing concentrations of isolated histone increased rickettsial infectivity, as opposed to competitively inhibiting Rickettsia-host cell association suggests that histones may act either as a direct rickettsial cellular entry mediator or as a bridging protein for rickettsial accumulation with an additional host cell surface protein. As previously noted, the C-terminal lysyl residues of histone $\mathrm{H} 2 \mathrm{~B}$ serve as a plasminogen recognition binding site (Das et al., 2007; Herren et al., 2006; Miles et al., 1991). In addition, the host plasminogen was reported as the dissemination/invasion activator for other tick-borne pathogens, i.e. Borrelia burgdorferi (Coleman et al., 1997) and Borrelia hermsii (Rossmann et al., 2007). Although the histone-plasminogen interaction occurs in a vertebrate infection system, further research should determine if additional arthropod-derived factors are involved.

Consistent with the current study, arthropod-derived histone has also been associated with other obligate intracellular bacteria closely related to Rickettsia. Wolbachia aggregates with Drosophila histone protein during spermegg cytoplasmic incompatibility, facilitating maternal transmission (Poinsot et al., 2003). It is not clear if rickettsiae manipulate the reproductive function in bloodfeeding arthropods; however, it is reasonable to suggest that two closely related organisms such as Wolbachia and Rickettsia, employing the same survival strategy (i.e. vertical transmission by arthropods), would utilize a conserved molecule such as histone. The ability of organisms such as Rickettsia to persist in nature via the perpetual infection of a variety of blood-feeding arthropods is testimony to a successful approach to survival in the absence of a complex amplification-dependent transmission cycle. As the specific mechanisms of infection become clearer, the selection of a ubiquitous and abundant molecule such as histone would be a viable approach for Rickettsia to continue to spread itself to a variety of host cell types. Continued studies on the infection mechanisms of arthropod hosts by Rickettsia will facilitate a better understanding of the ecology and epidemiology of vectorborne rickettsial diseases.

\section{ACKNOWLEDGEMENTS}

We thank T. Kurtti and U. Munderloh (UMN) for the ISE6 cell line and M. Kearney for statistical analysis. We also thank J. Macaluso, P. Sunyakumthorn, W. Pornwiroon and C. David (LSU/SVM) for technical assistance and advice. This research was supported by the Louisiana Board of Regents (LEQSF), the National Institutes of Health (P20 RR0201595), and the National Institutes of Allergy and Infectious Disease.

\section{REFERENCES}

Amsel, A. D., Rathaus, M., Kronman, N. \& Cohen, H. Y. (2008). Regulation of the proapoptotic factor Bax by Ku70-dependent deubiquitylation. Proc Natl Acad Sci U S A 105, 5117-5122.

Anacker, R. L., Mann, R. E. \& Gonzales, C. (1987). Reactivity of monoclonal antibodies to Rickettsia rickettsii with spotted fever and typhus group rickettsiae. J Clin Microbiol 25, 167-171.

Baake, M., Doenecke, D. \& Albig, W. (2001). Characterisation of nuclear localisation signals of the four human core histones. $J$ Cell Biochem 81, 333-346.

Behrens, S., Maier, R., de Cock, H., Schmid, F. X. \& Gross, C. A. (2001). The SurA periplasmic PPIase lacking its parvulin domains functions in vivo and has chaperone activity. EMBO J 20, 285-294.

Bolton, S. J. \& Perry, V. H. (1997). Histone H1; a neuronal protein that binds bacterial lipopolysaccharide. J Neurocytol 26, 823-831.

Borun, T. W., Scharff, M. D. \& Robbins, E. (1967). Rapidly labeled, polyribosome-associated RNA having the properties of histone messenger. Proc Natl Acad Sci U S A 58, 1977-1983.

Coleman, J. L., Gebbia, J. A., Piesman, J., Degen, J. L., Bugge, T. H. \& Benach, J. L. (1997). Plasminogen is required for efficient dissemination of $B$. burgdorferi in ticks and for enhancement of spirochetemia in mice. Cell 89, 1111-1119.

Das, R., Burke, T. \& Plow, E. F. (2007). Histone H2B as a functionally important plasminogen receptor on macrophages. Blood 110, 37633772 .

de la Fuente, J., Blouin, E. F., Manzano-Roman, R., Naranjo, V., Almazan, C., Perez de la Lastra, J. M., Zivkovic, Z., Jongejan, F. \& Kocan, K. M. (2007). Functional genomic studies of tick cells in response to infection with the cattle pathogen, Anaplasma marginale. Genomics 90, 712-722.

Emelyanov, V. V. \& Demyanova, N. G. (1999). Nucleotide sequence of the gene and features of the major outer membrane protein of a virulent Rickettsia prowazekii strain. Biochemistry (Mosc) 64, 494-503.

Featherstone, C. \& Jackson, S. P. (1999). Ku, a DNA repair protein with multiple cellular functions? Mutat Res 434, 3-15.

Gasteiger, E., Hoogland, C., Gattiker, A., Duvaud, S., Wilkins, M. R., Appel, R. D. \& Bairoch, A. (2005). Protein identification and analysis tools on the ExPASY server. The Proteomics Protocols Handbook, pp. 571-607. Edited by J. M. Walker. Totowa, NJ: Humana Press.

Hackstadt, T. (1996). The biology of rickettsiae. Infect Agents Dis 5, 127-143.

Heinzen, R. A., Hayes, S. F., Peacock, M. G. \& Hackstadt, T. (1993). Directional actin polymerization associated with spotted fever group rickettsia infection of Vero cells. Infect Immun 61, 1926-1935.

Heinzen, R. A., Grieshaber, S. S., Van Kirk, L. S. \& Devin, C. J. (1999). Dynamics of actin-based movement by Rickettsia rickettsii in Vero cells. Infect Immun 67, 4201-4207.

Herren, T., Burke, T. A., Das, R. \& Plow, E. F. (2006). Identification of histone $\mathrm{H} 2 \mathrm{~B}$ as a regulated plasminogen receptor. Biochemistry 45, 9463-9474. 
Holers, V. M. \& Kotzin, B. L. (1985). Human peripheral blood monocytes display surface antigens recognized by monoclonal antinuclear antibodies. J Clin Invest 76, 991-998.

Kedes, L. H. (1979). Histone genes and histone messengers. Annu Rev Biochem 48, 837-870.

Khan, I. U., Wallin, R., Gupta, R. S. \& Kammer, G. M. (1998). Protein kinase A-catalyzed phosphorylation of heat shock protein 60 chaperone regulates its attachment to histone $2 \mathrm{~B}$ in the $\mathrm{T}$ lymphocyte plasma membrane. Proc Natl Acad Sci U S A 95, 1042510430.

Kurtti, T. J., Mattila, J. T., Herron, M. J., Felsheim, R. F., Baldridge, G. D., Burkhardt, N. Y., Blazar, B. R., Hackett, P. B., Meyer, J. M. \& Munderloh, U. G. (2008). Transgene expression and silencing in a tick cell line: a model system for functional tick genomics. Insect Biochem Mol Biol 38, 963-968.

Li, H. \& Walker, D. H. (1998). rOmpA is a critical protein for the adhesion of Rickettsia rickettsii to host cells. Microb Pathog 24, 289298.

Lundemose, A. G., Kay, J. E. \& Pearce, J. H. (1993). Chlamydia trachomatis Mip-like protein has peptidyl-prolyl cis/trans isomerase activity that is inhibited by FK506 and rapamycin and is implicated in initiation of chlamydial infection. Mol Microbiol 7, 777-783.

Macaluso, K. R., Mulenga, A., Simser, J. A. \& Azad, A. F. (2003). Differential expression of genes in uninfected and Rickettsia-infected Dermacentor variabilis ticks as assessed by differential-display PCR. Infect Immun 71, 6165-6170.

Macaluso, K. R., Mulenga, A., Simser, J. A. \& Azad, A. F. (2006). Characterization of Dermacentor variabilis molecules associated with rickettsial infection. Ann N Y Acad Sci 1078, 384-388.

Martinez, J. J. \& Cossart, P. (2004). Early signaling events involved in the entry of Rickettsia conorii into mammalian cells. J Cell Sci 117, 5097-5106.

Martinez, J. J., Seveau, S., Veiga, E., Matsuyama, S. \& Cossart, P. (2005). Ku70, a component of DNA-dependent protein kinase, is a mammalian receptor for Rickettsia conorii. Cell 123, 1013-1023.

Marzluff, W. F. \& Duronio, R. J. (2002). Histone mRNA expression: multiple levels of cell cycle regulation and important developmental consequences. Curr Opin Cell Biol 14, 692-699.

Miles, L. A., Dahlberg, C. M., Plescia, J., Felez, J., Kato, K. \& Plow, E. F. (1991). Role of cell-surface lysines in plasminogen binding to cells: identification of alpha-enolase as a candidate plasminogen receptor. Biochemistry 30, 1682-1691.

Mulenga, A., Macaluso, K. R., Simser, J. A. \& Azad, A. F. (2003). Dynamics of Rickettsia-tick interactions: identification and characterization of differentially expressed mRNAs in uninfected and infected Dermacentor variabilis. Insect Mol Biol 12, 185-193.

Munderloh, U. G. \& Kurtti, T. J. (1989). Formulation of medium for tick cell culture. Exp Appl Acarol 7, 219-229.

Munderloh, U. G. \& Kurtti, T. J. (1995). Cellular and molecular interrelationships between ticks and prokaryotic tick-borne pathogens. Annu Rev Entomol 40, 221-243.

Poinsot, D., Charlat, S. \& Mercot, H. (2003). On the mechanism of Wolbachia-induced cytoplasmic incompatibility: confronting the models with the facts. Bioessays 25, 259-265.

Pornwiroon, W., Pourciau, S. S., Foil, L. D. \& Macaluso, K. R. (2006). Rickettsia felis from cat fleas: isolation and culture in a tick-derived cell line. Appl Environ Microbiol 72, 5589-5595.

Pornwiroon, W., Bourchookarn, A., Paddock, C. D. \& Macaluso, K. R. (2009). Proteomic analysis of Rickettsia parkeri strain portsmouth. Infect Immun 77, 5262-5271.
Prabhakar, B. S., Allaway, G. P., Srinivasappa, J. \& Notkins, A. L. (1990). Cell surface expression of the $70-\mathrm{kD}$ component of $\mathrm{Ku}, \mathrm{a}$ DNA-binding nuclear autoantigen. J Clin Invest 86, 1301-1305.

Qiu, H. \& Wang, Y. (2008). Quantitative analysis of surface plasma membrane proteins of primary and metastatic melanoma cells. J Proteome Res 7, 1904-1915.

Ramakrishnan, V. G., Aljamali, M. N., Sauer, J. R. \& Essenberg, R. C. (2005). Application of RNA interference in tick salivary gland research. J Biomol Tech 16, 297-305.

Reif, K. E. \& Macaluso, K. R. (2009). Ecology of Rickettsia felis: a review. J Med Entomol 46, 723-736.

Rossmann, E., Kraiczy, P., Herzberger, P., Skerka, C., Kirschfink, M., Simon, M. M., Zipfel, P. F. \& Wallich, R. (2007). Dual binding specificity of a Borrelia hermsii-associated complement regulatoracquiring surface protein for factor $\mathrm{H}$ and plasminogen discloses a putative virulence factor of relapsing fever spirochetes. J Immunol 178, 7292-7301.

Silverman, D. J. \& Wisseman, C. L., Jr (1979). In vitro studies of rickettsia-host cell interactions: ultrastructural changes induced by Rickettsia rickettsii infection of chicken embryo fibroblasts. Infect Immun 26, 714-727.

Simser, J. A., Mulenga, A., Macaluso, K. R. \& Azad, A. F. (2004). An immune responsive factor $\mathrm{D}$-like serine proteinase homologue identified from the American dog tick, Dermacentor variabilis. Insect Mol Biol 13, 25-35.

Sunyakumthorn, P., Bourchookarn, A., Pornwiroon, W., David, C., Barker, S. A. \& Macaluso, K. R. (2008). Characterization and growth of polymorphic Rickettsia felis in a tick cell line. Appl Environ Microbiol 74, 3151-3158.

Tai, Y. T., Podar, K., Kraeft, S. K., Wang, F., Young, G., Lin, B., Gupta, D., Chen, L. B. \& Anderson, K. C. (2002). Translocation of Ku86/Ku70 to the multiple myeloma cell membrane: functional implications. Exp Hematol 30, 212-220.

Uchiyama, T., Kawano, H. \& Kusuhara, Y. (2006). The major outer membrane protein rOmpB of spotted fever group rickettsiae functions in the rickettsial adherence to and invasion of Vero cells. Microbes Infect 8, 801-809.

Watson, K., Gooderham, N. J., Davies, D. S. \& Edwards, R. J. (1999). Nucleosomes bind to cell surface proteoglycans. J Biol Chem 274, 21707-21713.

Weiss, E. (1982). The biology of rickettsiae. Annu Rev Microbiol 36, 345-370.

Whitworth, T., Popov, V. L., Yu, X. J., Walker, D. H. \& Bouyer, D. H. (2005). Expression of the Rickettsia prowazekii pld or tlyC gene in Salmonella enterica serovar Typhimurium mediates phagosomal escape. Infect Immun 73, 6668-6673.

Zanettii, A. S., Pornwiroon, W., Kearney, M. T. \& Macaluso, K. R. (2008). Characterization of rickettsial infection in Amblyomma americanum (Acari: Ixodidae) by quantitative real-time polymerase chain reaction. J Med Entomol 45, 267-275.

Zavala-Castro, J. E., Small, M., Keng, C., Bouyer, D. H., ZavalaVelazquez, J. \& Walker, D. H. (2005). Transcription of the Rickettsia felis ompA gene in naturally infected fleas. Am J Trop Med Hyg 73, 662-666.

Zhu, G., Chen, H., Choi, B. K., Del, P. F. \& Schifferli, D. M. (2005). Histone $\mathrm{H} 1$ proteins act as receptors for the 987P fimbriae of enterotoxigenic Escherichia coli. J Biol Chem 280, 23057-23065.

Zlatanova, J. S., Srebreva, L. N., Banchev, T. B., Tasheva, B. T. \& Tsanev, R. G. (1990). Cytoplasmic pool of histone H1 in mammalian cells. J Cell Sci 96, 461-468.

Edited by: J. Renaudin 